\title{
Virtual Motion capture technology Applied In assist-training system of Sports
}

\author{
Fabin Wang \\ Department of Physical Education, Gansu Normal University for Nationalities, Hezuo 747000, China \\ zfx6289@163.com
}

Keywords: Virtual simulation; Sports training; computer information technology.

\begin{abstract}
The application of simulation technology in the field of sports is used wildly, which is combined closer and closer with sports skills and tactics. It is an important supplement method for sports training. Constructing the virtual simulation of movement by means of computer information technology, putting virtual simulation technology into the application of training. This paper explored the application of virtual simulation technology in sports training through applying the method of virtual simulation technology to aid sports training. This system is beneficial for acquiring knowledge and skills, realizing the effective organization and management for training information, which can provide a good training environment for sports training.
\end{abstract}

\section{Introduction}

Motion Capture is to record the motion track in three dimensions by sensor devices, to transform it to motion data, and then to drive the virtual human to move based on these data. In recent years, there are a lot of works done on the Motion Capture outside nation and many systems on Motion Capture have been provided to use. But all of the systems are so expensive that few institutes or companies in our country can afford. A few institutes in our country have been engaging in the business for several years, however, the images collected by the system made by them are often planar and have no three-dimensional motion parameter because the images are captured by cameras and have to be disposed on sensibility experience. As a result, it is very necessary to design a 3D Motion Capture system by ourselves to fulfill the internal demand.

Simulation technology is a real system model built by the use of computer technology, which is a method for having a simulated study. It is a comprehensive experimental technology based on the system science, system identification, control theory, computing technology and control engineering. It is applied to the field of sports by computer simulation technology, coach's training intention, manager's organizing schemes, as well as the athletes' training process, so as to explain the sports system with analyzing, forecasting, and evaluating, which is an experimental subject. The key technique of simulation technology is mainly about mathematical modeling and the acquisition of motion data. Virtual reality and simulation technology is playing a more and more important role, which can provide a broader space for the application of high-tech in the sports field. As the master of nature and human society, modeling the human body itself is in a very important position, which has become a hot issue in human engineering, computer graphics, artificial life and biomechanics study.

\section{The Principles of Constructing assist-training system of Sports}

With a further recognition on the experimental features of economic management, we have a deep thinking about the principles of constructing assist-training system, and summarize the following principles:

1. Systemic Principle. Due to involving many disciplines and covering many majors, the construction of sports assist-training system is a complex systemic project. In order to build a complete and reasonable system, we must follow the system theory, especially the points of view about the integrity, the interrelation, and the dynamic evolution. In addition to the orderly planning and integration among the knowledge in the assist-training system, the assist-training system must be 
combined with other practice training, such as theory training, teachers' scientific researches, and advantages of school disciplines and the development of local economy.

2. Modular Principle. The assist-training system of sports should include the training of computer software and hardware technique, database technology, data acquisition technology, information retrieval, data mining technology, artificial intelligence technology, simulation technology and other disciplines and relevant professional experiment training. According to the objective differences among contents of various disciplines and majors and the unified planning based on the disciplines, breaking traditional methods based on courses, the system divides the integrity into modules scientifically and reasonably, therefore the students of different disciplines and different majors can choose related modules to learn. In the process of dividing the modules, we should abide by the principles of high aggregation and low coupling of module contents to fully embody the differences and relations among disciplines.

3Hierarchy Principle. In addition to making a lateral distinction, according to the different training aims and the deep analysis of assist-training system, we should map out different vertical hierarchies scientifically and reasonably, and build a hierarchical and gradual assist-training system system to meet different learning needs of students in different grades, different periods and different purposes.

4. Profitability Principle. No matter how to divide horizontally or vertically, the standard to check the assist-training system is reasonable is benefit. There are three aspects of benefit: first, it is the technical benefit to meet the talent training target; second, it is the economic benefit under the software and hardware environment; the last one is the social benefit that can demonstrate, radiate and lead the development. If the three aspects are noticed, the integration and completion of constructing sports assist-training system can be guaranteed.

\section{The Motion Capture of Motion Information Technology}

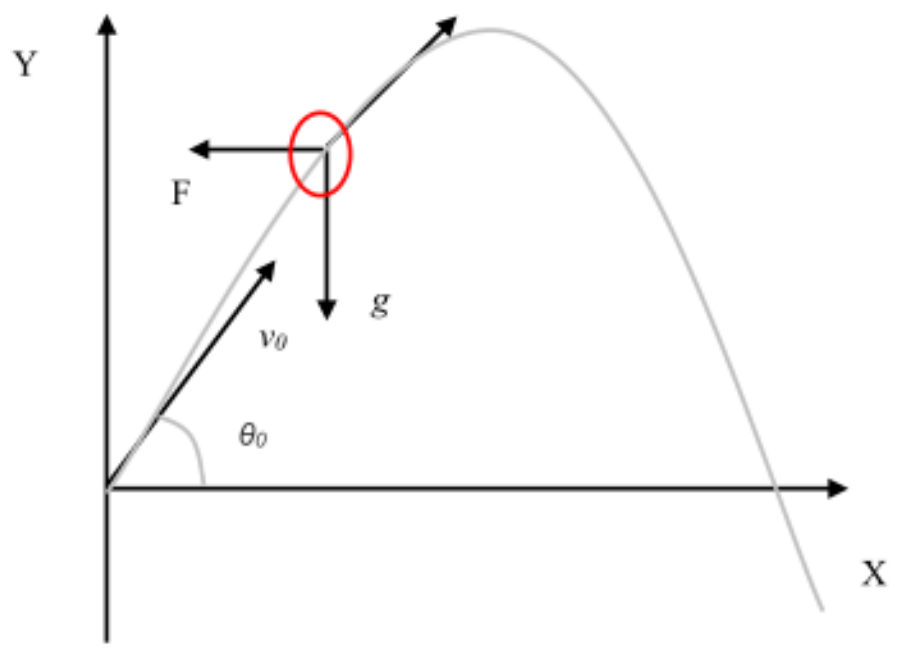

Fig. 1 Parabolic Motion

The motion capture technology is a new high technology for capturing the athlete's skill tactics and cooperation, the real-time 3D trajectory of the moving object and making digital analysis by using the motion capture system. The motion capture system is a kind of high technology equipment for the accurate measurement of the condition of the moving object in 3D space, its working principle is based on computer graphics, by arranging a number of video capturing devices to record the condition of the moving objects (tracker) in the form of image, processed by the computer, getting the spatial coordinates (X, Y, Z) at different time unit of the different objects (tracker). Through analyzing the force of gravity and the air resistance, the flight trajectory of basketball is a parabola. The parabola trajectory of basketball and the final landing point is depended on the initial velocity vector that fingers of athletes left basketball, including velocity and direction. Using $X^{\prime}, Y$ and $Z$ as $3 D$ coordinate to describe the motion model of basketball. The $X^{\prime}$ axis represents the horizontal direction 
from hand to basketball rack, $Y$ axis represents the moving height of basketball. It can be assumed that the deflexion-angle of $X^{\prime}$ axis and the direction of the actual shooting in $X^{\prime} Z$ plane is $a$. Assuming $X_{k}$ axis is the projection of basketball's flying parabolic path in $X^{\prime} Z$ plane. The following is the analysis on $X_{k} Y$, which is as shown in Fig. 1:

According to the characteristics of the athletes' posture parameters to estimate the inertial parameters of human body, determining the rotational inertia parameters and other individual parameters needed by the Newton Euler model, acquiring the equations of personalized motion, according to the equation to prove the rationality of the new motion $(\mathrm{t})$. As shown in Fig 2, through the interaction process of the visualized interaction design, motion validation and feedback, it can complete 3D simulation design as the breakthrough technology.

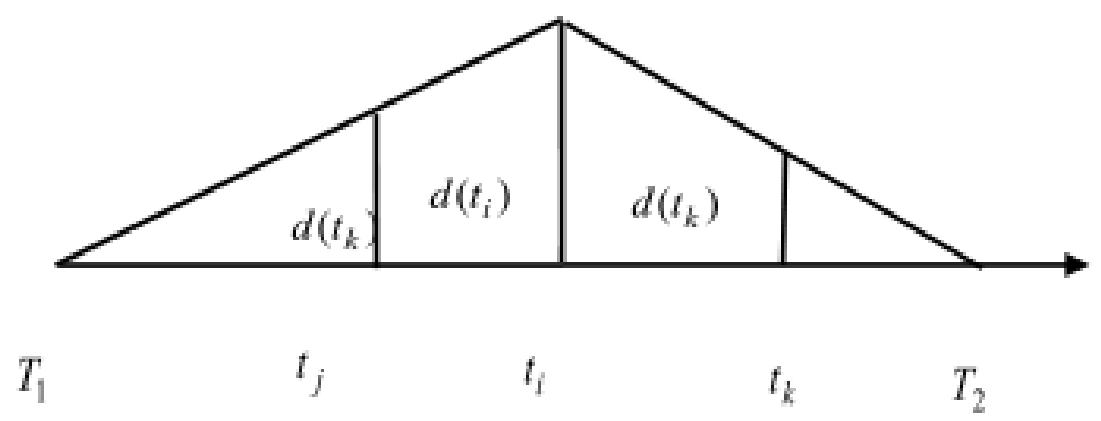

Fig.2 The Tectonic of the Offset

Motion capture technology can catch the action of the basketball players, which is easy to make quantitative analysis, combined with human physiology, principles of physics, to study the researching method, making the physical training getting rid of the pure experience, so as to enter into the scientific and digital age. Recording the poor performance of the athletes' compared with the performance of the elite athletes, so as to help their training, providing the accurate quantitative basis for the scientific training. With the aid of the motion capture technology and computer image processing technology, it can greatly improve the visibility of the basketball technical training, which is helpful for the quantitative analysis of technical action, displaying the results graphically, including the displacement, velocity, force, etc. Based on it, it made deep analysis about the "ideal" action as well as the athlete's technical movement, putting forward the guiding advice for the athletes to improve the technique action.

Through the training and comparison of virtual simulation technology, teachers can also make evaluation for the athlete's skills and point out the deficiency.

\section{Conclusion}

Introducing the virtual simulation technology can improve the training environment, expand the training capacity, and adapt itself to P.E. training and sports training as well as the needs of the reform to develop the virtual simulation technology and aid sports training, it is no longer confined to the tangible fixed place, the training space and training time and space can be got invisible expansion, which also can promote the self-learning, self-analysis, self-training developed in the form of the "three self", through the virtual simulation technology, it can expand the means of sports training and methods of sharing resources.

\section{References}

[1]Chen,J., Basketball technique of multimedia database development and open. Journal of Shanghai sports institute, 29-31(2007).

[2]Wang, H. G., The principle and application of computer simulation. national defense university of science and technology press, (2004). 
[3]Mary, A., The application of computer simulation technology in the field of sports training.Sports scientific research, 68-70(2008).

[4]Wang, H.G., The principle and application of computer simulation. national defense university of science and technology press . (2004).

[5]Williamson, O., Computer simulation technology applied in the field of sports training. Sports research. 68-70 (2008). 